\title{
Design of integrated Ka-band reflective phased array antenna element
}

\author{
Anatoly I. Firsenkov ${ }^{1}$, Anton B. Guskov ${ }^{1}$, Alexander S. Smirnov ${ }^{1}$, \\ Vladimir M. Krekhtunov ${ }^{2}$, and Elena $V$. Komissarova ${ }^{2^{*}}$ \\ ${ }^{1}$ Public Corporation "Plant "Magneton" 9, Kurchatova str., Saint-Petersburg,194223, Russia \\ ${ }^{2}$ Bauman Moscow State Technical University, 5 building 1, $2^{\text {nd }}$ Baumanskaya str., Moscow, 105005 , \\ Russia
}

\begin{abstract}
The results of a constructively simple high-tech small-sized integrated element of the Ka-band electric beam scanning reflective phased array antenna (PAA) development are shown. Beam scanning sector of the phased array antenna is up to 60 by both coordinates. The PAA element is based on the Faraday type waveguide ferrite phase shifter, which works on the circular polarized electromagnetic waves. More than 30000 PAA elements has been produced, $100 \%$ control and statistical data processing of their main characteristics has been carried out. Graph for initial phases and steepness of linearized phase characteristics, medium and maximum losses of PAA elements are performed. Average value of the average deviation for parameters which is important for PAA and the beam control system design have been determined. The developed element can be used in PAA which have different sizes, forms and sectors of electrical beam scanning. Small deviations in the characteristics of PAA elements from the linearized characteristics indicate the high quality of the production and their identity, which makes it possible to create PAA without taking into account the real individual parameters of mass-produced PAA elements.
\end{abstract}

\section{Introduction}

To the early 1970s, microwave radio technology in the world had made great strides in the development of ferritic microwave devices, in the study of waveguide-based array antenna, taking into account the mutual influence of PAA elements, PAA excitation systems and phase shifter's control systems $[1,2,3,4]$.

The beginning of particular microwave band PAA design for air defense systems can be dated to 1976, when work on the C300-PMU and Patriot systems began simultaneously in the USSR and the USA. Both systems had common things like purpose, waveguide ferrite phase shifters (WFPS) using, spatial excitation, monoimpulse swaddling, element by element phasing, aperture size, etc. The differences were type and switching speed of phase shifters, polarization type of emitted and received waves, range and bandwidth of working frequencies.

In centimetre and millimeter wavelengths multi-element phased array antennas with a number of elements more than $10^{3}$ are usually designed as pass or reflective type with spatial excitation [6]. Pass type PAA are characterized by a higher gain, lower side lobe level and the ability of separate optimization of the receiving and aperture array antennas.

${ }^{*}$ Corresponding author: $\underline{\text { me ken@list.ru }}$ 
However, during the disign of pass type wide-angle electric beam scanning PAA in the millimeter wavelength there are a lot of engineering and technological problems which are associated with the PAA elements close arrangement and the needs to set WFPS connection wires to the PAA side surface.

All named above charachteristics of reflective type PAA are worse than in pass type PAA but reflective type PAA have some advantage. First of all, their elements could be larger with the same PAA beam scanning sector. Secondly, the PAA beam control system could be installed behind the antenna, that's why the construction could be designed as module type which could be easy replaced in a case of breakage. So in a number of practical applications, the choice of reflective type PAA design is more preferable then the pass type.

\section{The rationale of WFPS scheme choice}

There are a lot of requirements to waveguide ferrite phase shifters for PAA elements such as:

-Working with electromagnetic waves with predetermined polarization;

-Small microwave losses;

-High switching speed and low remagnetization energy;

-Stability of the initial phase and phase shifting;

-"Reception-transmission" mode reciprocity;

-Phase characteristics' linearity with all level of the control signal;

-Small dimensions and mass;

-Processability of the construction;

-Good reproducibility in mass production conditions;

-Resistance to mechanical and climatic influences.

The most used types of phase shifters in integrated transmitting and receiving PAA elements are [2]:

1) WFPS based on rectangular waveguide with transversely magnetized ferrite toroid, it has high switching speed, but it's non-reciprocal, has complex construction and high remagnetization energy;

2) Regia-Spencer WFPS based on rectangular waveguide with longitudinally magnetized ferrite rod (FR) characterized by high "activity", low switching speed and narrowband;

3) Farraday type WFPS based on circle or rectangular waveguide with longitudinally magnetized FR, has simple and manufacturable construction. WFPS is working on waves with circular polarization of electromagnetic waves.

According to the totality of the above characteristics, the main requirements are best satisfied by the Faraday type WFPS. On their basis, it is possible to create PAA elements that transmite and receive electromagnetic waves with circular, linear and adjustable polarizations. The characteristics of the Faraday WFPS, and, first of all, switching speed and remagnetization energy, vary widely depending on the design.

Depending on the required switching speed, which is characterized by the WFPS remagnetization time from one phase state to another, the phase shifter can have different designs. Energy $W$, which is spent by control system for WFPS remagnetization connected to remagnetization time $t_{\mathrm{H}}$ as [6]

$$
W=W_{0}\left(1+\frac{t_{0}}{t_{H}}\right)
$$

Where $W_{0}$ - energy which is spent on nonmetalizied ferrite rod remagnetization, called «hysteresis loss»; $\mathrm{t}_{0}$ - characteristic time, depending of parameters of ferrite material: 
coercive force $\mathrm{H}_{c}$; residual magnetic induction $\mathrm{B}_{\mathrm{r}}$ и squareness ratio of hysteresis loop $\mathrm{K}_{\text {пр }}$, transverse dimensions and lengths of ferrite rod, thickness and conductivity of metallization on its lateral surfaces.

If lateral surfaces of ferrite rod are full metalized remagnitization time has maximal value and could be more than 100 microseconds for X-band WFPS. If ferrite rod of WFPS is partly metalized then switching speed increases of the WFPS increases, remagnitization time decreases down to several tens of microseconds [6]. Extremely high switching speed is achieved when WFPS is designed on the basis of longitudinally magnetized ferrite rod, which doesn't contain any metallization on its lateral surfaces. In this case remagnitization time could be decreased to value of toroidal non-reciprocal phase shifter, but it has a complex design and producing technology.

Since $70^{\text {th }}$ companies in USSR have accumulated extensive experience in the development, implementation and mass production of waveguide ferrite Faraday phase shifters, waveguide-dielectric radiators and integrated elements of phased array antennas designed to operate in the centimeter wave range. The most successful problem solution is described in [5,7], where were summarized the experience of development and mass production of PAA elements for the S-300 PMU radar system and described the technological operations, the quality and accuracy of which significantly affect on the characteristics of the waveguide ferrite phase shifters and PAA elements in general.

\section{The design of Ka-band PAA integrated elements}

At the beginning of 2000 real problem of the Ka-band radar system design was pointed [8], so PAA such as S300-PMU as well [7]. However, the full transfer of design and technological solutions developed in the creation of high-tech PAA elements of S300-PMU was impossible. Due to a decrease in the wavelength in the HF band, the dimensions of parts are not only proportionally reduced, but also the process of their manufacture and assembly of PAA elements becomes more complicated.

Creation of the element base for HF PAA, including ferrite phase shifters and integrated elements on their basis has a number of specific features. First of all, they include technological aspects related to the low mechanical strength of parts made of ferrites and dielectrics, the required high accuracy of their machining, as well as the complexity of the assembly of elements and the difficulties of its automation. Also, in contrast to the manufacture of X-band PAA elements, there are problems with the very close placement of elements in the antenna array and the output of the control system wires to side surface of a PAA.

The problem of Ka-band PAA element with Faraday type WFPS design was intensified at the beginning of 2004, when there was a real need to create multi-element passing PAA with spatial feeding and a wide-angle electrical beam scanning in sector $\pm 90^{\circ}$ in both coordinates [8]. It was successfully solved by joint efforts of employees of a number of enterprises [9].

Schemes and drafts of pass and reflective PAA elements are on Fig. 1a and $1 \mathrm{~b}$.

On Fig. 1: 1 - ferrite rod, 2 -longitudinally magnetization coil, 3 - magnetic conductor, 4 -waveguide transformer, 2 and 6 - transmitting and receiving waveguide-dielectric radiator.

On Fig. 2 draft of the integrated PAA element and its longitudinal section is shown. Position designations are accepted by [9].

PAA element consists of receiving 1 and transmitting 2 dielectric radiators and ferrite Faraday phase shifter, which includes longitudinally magnetization coil, installed inside magnetic conductor 11 on the square ferrite rod 7 with metallization of on its lateral surfaces 9 , between the ends of rod and the ends of the dielectric radiators matching dielectric 


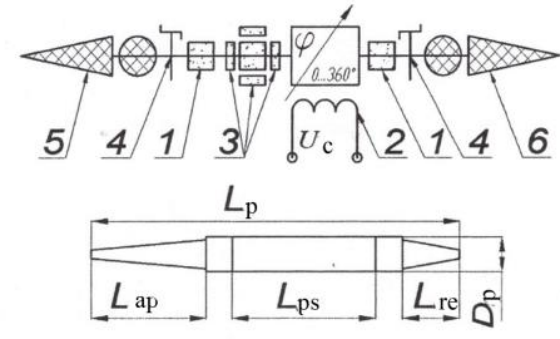

a

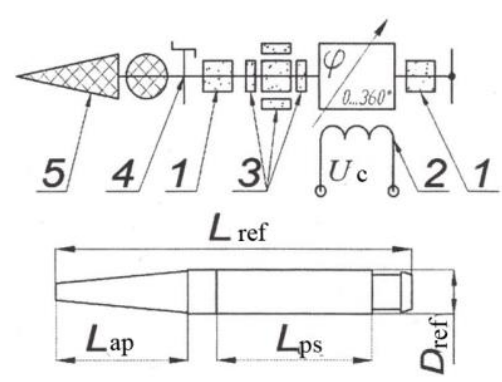

$\mathrm{b}$

Fig. 1. Schemes and draft of PAA elements: pass (a) and reflective (b).

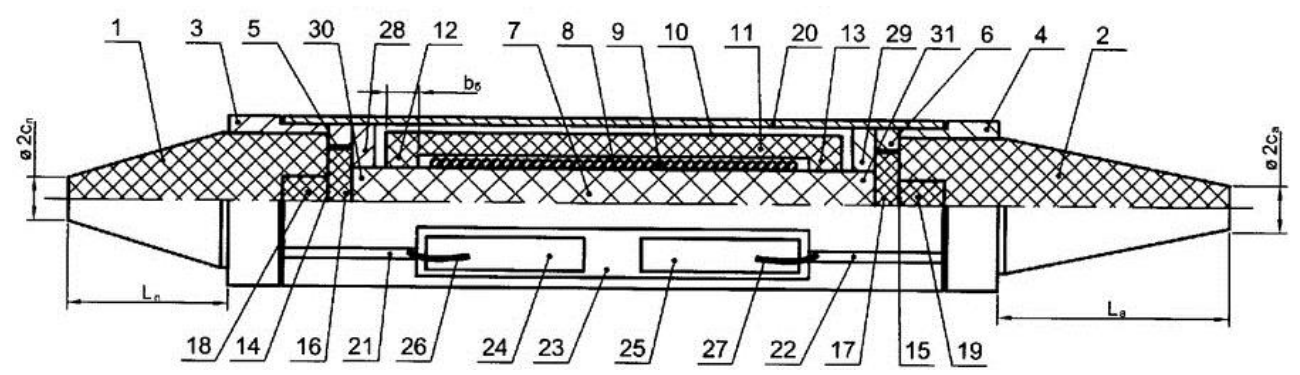

Fig. 2. Draft of the integrated PAA element.

inserts 5 and 17 are installed and placed together with the cylindrical shanks of the dielectric radiators and a ferrite rod inside the waveguide. The magnetic conductor is made in the form of U-shaped yokes 10, each of which contains a longitudinal plate 11 and two pads 12 and 13 adjacent to the side surface of the ferrite rod.

In PAA element construction normalized materials are used, for example magnetic conductors' parts are made from lithium ferrite 1SCH12 with low microwave losses and high rectangularity of hysteresis lope [20].

The constructor's documentation was elaborate for the practical realization of PAA element, which is shown on Fig. 2 and it was mass produced. Main characteristics of PAA elements are in Table 1.

Table 1. Characteristics of PAA elements.

\begin{tabular}{|c|c|}
\hline Characteristic & Value \\
\hline Fraquency range & Ka \\
\hline Transverse size / length of the phase shifter in wavelength $\lambda$ & $0,51 / 2,3$ \\
\hline Inter-element distance $\mathrm{d}_{\Delta}$, in wavelength $\lambda$ & 0,68 \\
\hline Maximum losses, less then, $\mathrm{dB}$ & 1,5 \\
\hline Phase shear, more then, $^{\circ}$ & 400 \\
\hline Phase deviation, less then, $^{\circ}$ & 7 \\
\hline Phase shifter's remagnetization time, less then, $\mu \mathrm{s}$ & 100 \\
\hline Phase shifter's remagnetization energy, less then, $\mu \mathrm{J}$ & 100 \\
\hline
\end{tabular}

Based on pass PAA elements [9] worked on circle polarized waves, reflective PAA element with linear polarization of electromagnetic waves was designed [10]. It became possible after instalation of quadrupole tuned polarizer with magnetic shield inside WFPS without any changes of transversal dimentions, which decreeses reciprocal magnetic field influence. 


\section{Retlective PAA integrated elements}

The development of reflective PAA with spatial feeding and their element base until recently received insufficient attention from designers.

In [11] and [12] considered the technical problems that were solved with the creation of the millimeter wave range PAA with electric beam scanning in a conic sector of $2 \theta_{\mathrm{s}}=50^{\circ}$. Antenna array contains 3,600 elements and has a diameter of $2 \mathrm{R}=70 \lambda$. Structurally, the PAA consists of subarrays with 88 and 118 PAA elements. Inasmuch as large diameter of phased array antenna elements (inter-elements interval is about $d_{\Delta}=1,1 \lambda$ ) designers had was available to construct PAA with narrow beam scanning sector. The consequence of this was the presence of side main lobes in the radiation pattern (RP).

Later, as a result of increased interest to reflective PAA, a number of publications on their element base appeared. In [13] and [14] proposed schemes for constructing WFPS, recircularly polarized waves reflected. However, judging by the descriptions, it is problematic to build high-tech integrated PAA elements suitable for mass production on their basis.

Some progress in this direction has been achieved in recent years [15...18]. In [15] questions about the construction of multi-element modular PAA were discussed, the mutual influence of radiators was analyzed and the problems of measuring the phase characteristics of PAA elements in the antenna array were solves. PAA contains about $4 \cdot 10^{4}$ elements installed in an interval $d_{\Delta}=0,65 \lambda$, when the diameter of the aperture is about $136 \lambda$ This provides the beam scanning sector $\pm 45^{\circ}$. However, the issues of PAA integrated elements construction are not disclosed in the article.

According to [19], the problem of creating a high-tech small-sized reflective PAA element which could be operated on electromagnetic waves with circular polarization has been successfully solved. Item reflective PAA operates in the frequency band $(34 \pm 0,5)$ $\mathrm{GHz}$ and has features: adjustable phase shift - at least $400^{\circ}$; losses are not more than 1.5 $\mathrm{dB}$; remagnitization time - not more than $100 \mu \mathrm{s}$; operating temperature range: from -40 to $+55^{\circ} \mathrm{C}$; diameter and length of the RPAA element- $5.8 \mathrm{~mm}$ and $47.7 \mathrm{~mm}$, respectively.

A topical task is to reduce the diameter of the PAA element in order to expand the sector of electric beam scanning and ensure the identity of commercially produced PAA elements in terms of phase-time characteristics and insertion losses.

To that end PAA element was upgraded. On Fig. 3 the draft of upgraded reflective PAA element described in [16] is shown. On Fig. 3: 1 - dielectric radiation rod; 2 - radiator's waveguide; 3 - matching waveguide; 4 - matching dielectric transformer; 5 - connective waveguide; 6 - ferrite rod; 7 - conductive coating of the ferrite rod side surface; 8 magnetization coil; 9 - ferrite magnetic yoke; 10 - pad of ferrite yoke; 11 - conductive coating of the ferrite rod back surface; 12 - phase shifter's thin metal body; 13 - dielectric plug; 14 magnetization coil wire.

In upgraded reflective PAA element construction normalized materials are used. In particular for the manufacture of ferrite rod and magnetic cores are also used ferrite brand 1SCH12 [20] having low microwave losses and a high squareness of the hysteresis loop. For the manufacture of radiators and matching transformer used specially designed composite dielectrics [20] with the required value of dielectric permeability $(\varepsilon=4 \ldots 10)$. Using of such dielectrics allows to apply a casting technology that provides high electrical characteristics of the PAA elements together with a low cost of dielectric parts.

While designing of the integrated reflective PAA element, special attention was paid to the creation of the ferrite rod magnetization system with low scattering losses. As a result of optimization of the thickness of the conductive coating of the ferrite rod side surface, the choice of the shape and size of ferrite magnetic core pads and method of its fixing on the ferrite rod side surface, has a high linearity of phase-time characteristics (PTC) is realized with volt-second method of controlling the phase shifter, which is described by the expression 


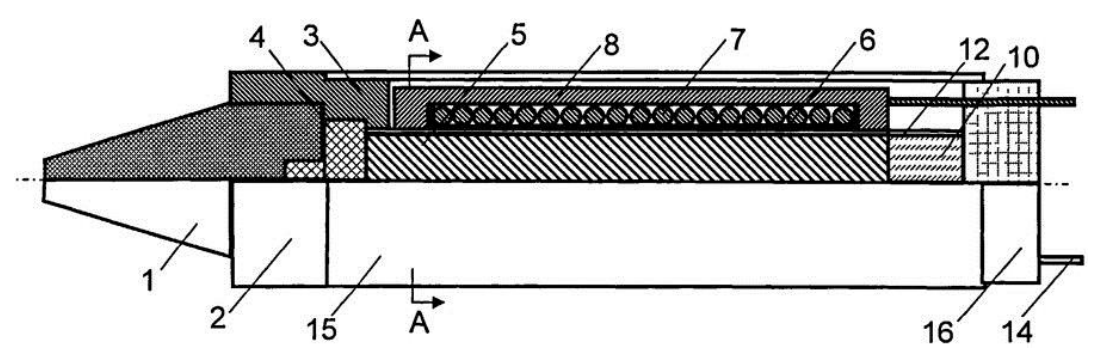

Fig. 3. Reflective PAA element draft.

$$
\Delta \varphi(t)=\Delta \varphi_{0}+K_{\varphi} t
$$

here $\Delta \varphi_{0}$ - the initial phase shift produced by the phase shifter at the duration of the magnetization pulse $t=0 ; K_{\varphi}$ - the steepness of the linearized PTC. These parameters were recorded to computer memory of control system and were used for calculations of phase shifters beam scanning pusles.

According to the developed design and technological documentation, a batch of PAA elements with a total number of elements more than $3 \cdot 10^{4}$ was released, their experimental characteristics were carried out with $100 \%$ control of the parameters: introduced microwave losses and phase characteristics. As a result of statistical processing of measurement results, it was found that the numerical values of the above parameters are distributed according to the normal law.

On Fig.4 the linearized PTC of Faraday type waveguide ferrite phase shifter is shown.

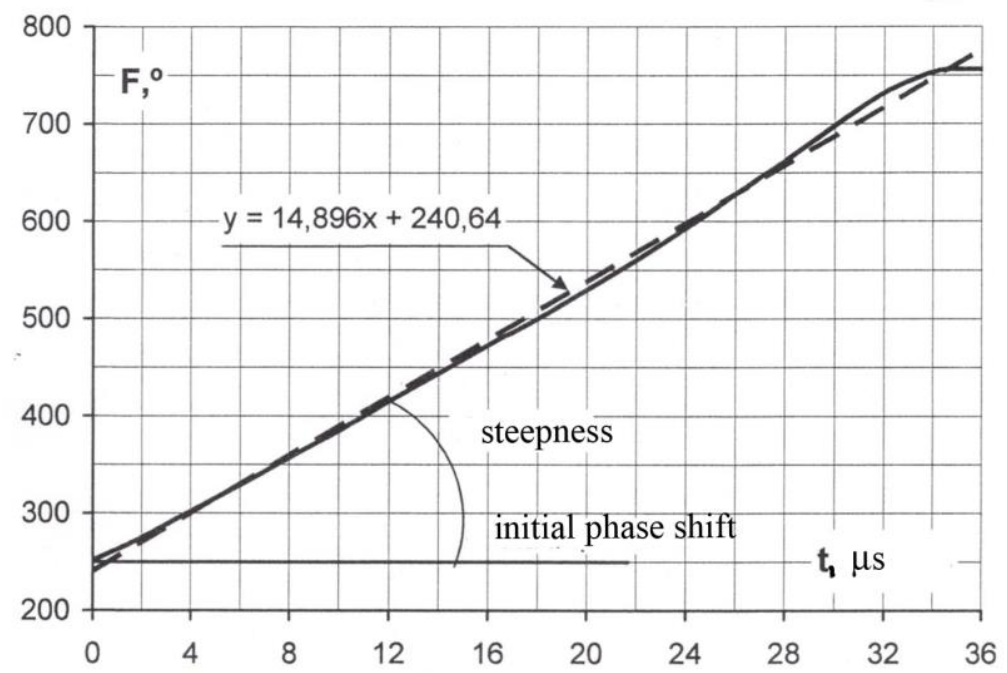

Fig. 4. Linearized phase-time characteristic of Faraday type WFPS.

Solid line here is the average PTC, which was found as the result of experimental researches of manufactured phase shifters, the dashed line is the linearized PTC.

The results of statistical processing of experimental data:

- the average initial phase shift value is $\Delta \varphi_{0}=240,64^{\circ}$, with deviation of initial phase shift value $17,76^{\circ}$;

- the average steepness of the linearized PTC is $\mathrm{K}_{\varphi}=14,84 \% / \mu \mathrm{s}$ with deviation $0,37 \% / \mu \mathrm{s}$;

- the average insertion loss is $1,05 \mathrm{~dB}$ with deviation $-0,1 \mathrm{~dB}$;

- the maximum insertion loss is $1,27 \mathrm{~dB}$ with deviation $0,14 \mathrm{~dB}$. 
The nonlinearity of the PTC of real integrated reflective PAA elements leads to errors in the phase distribution across the PAA aperture, therefore the level of side lobes of antenna array radiation pattern increases and its directional coefficient $\mathrm{D}_{0}$ decreases. Phase errors correlated on a large base of PAA aperture are especially dangerous. They can occur if the phase shifters have systematic smooth nonlinearities. To clarify this circumstances averaging of phased array elements PTC was conducted with pre-sorting by the mean of square deviation and dividing into groups of 4,000 elements For each group average PTC was graphed and the average values of $\Delta \varphi_{0}$ and $K_{\varphi}$ were calculated

If phase errors are a stationary variable with zero average mean and known variance value $\sigma^{2}$, and the correlation errors radius is $\rho_{0}$ that is the antenna array step $d_{\Delta}$, so, the average antenna array radiation pattern can be calculated as [6]:

$$
|\overline{F(\theta, \psi)}|^{2}=\exp \left(-\sigma^{2}\right) \cdot\left[F_{0}^{2}(\Theta, \psi)+\sigma^{2} \frac{4 \pi^{2} \rho_{0}^{2}}{D_{0} \lambda^{2}} \exp \left(-\frac{\pi^{2} \rho_{0}^{2} \sin ^{2} \theta}{\lambda^{2}}\right)\right],
$$

where $F_{0}(\theta, \psi)$ - antenna array radiation pattern, a $\mathrm{D}_{0}$ - antenna array directional coefficient without any phase errors.

The first factor (3) shows the reduction of the antenna array directional coefficient. The second term gives the background level of the side lobes outside the main area and the side lobes closest to it. It's interesting to find out what are these parameters if we assume that all PAA elements are different, but with a linear PTC as well, if all of the PAA elements have the same PTC steepness, but different initial phase, or if all the manufactured PAA elements have the same PTC.

Average standard deviation for these three cases are $7,5^{\circ}, 11^{\circ}$ and $16^{\circ}$ accordingly. As the results of analysis was found out that PAA elements identity so highly, that elements may be used to design PAA without any individual information about $\Delta \varphi_{0}$ and $K_{\varphi}$.

For PAA with numbers of elements $\mathrm{N}=4 \cdot 10^{4}$ reduction of the antenna array directional coefficient and background level were calculated. Correlation errors radiuswas set as

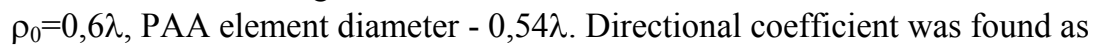

$$
D=v \cdot \frac{4 \pi N \rho_{0}^{2}}{\lambda^{2}}
$$

where $\mathrm{N}$ - number of PAA elements; $v$ - aperture coefficient was taken as $v=0,3$.

The calculation results are in table 2 .

The experimental researches result shows a possibility to use the producted PAA element in different sized PAA without any individual tuning. In this case background level will rise on $6 \mathrm{~dB}$ only and this value will be lower on $20 \mathrm{~dB}$ in comparison with a value of far side lobes (about $-30 \ldots-40 \mathrm{~dB}$ ).

Table 2. Radiation pattern parameters of PAA.

\begin{tabular}{|c|c|c|}
\hline $\begin{array}{c}\text { Average standard } \\
\text { deviation, }\end{array}$ & $\exp \left(-\boldsymbol{\sigma}^{\mathbf{2}}\right) \mathbf{d B}$ & $\begin{array}{c}\text { Background } \\
\text { level, dB }\end{array}$ \\
\hline 7,5 & 0,07 & $-68,7$ \\
\hline 11 & 0,16 & $-65,4$ \\
\hline 16 & 0,34 & $-62,1$ \\
\hline 30 & 1,2 & $-56,2$ \\
\hline
\end{tabular}




\section{Conclusion}

1. High-tech small-size integrated reflective type PAA element is designed. It works in PAA with eletrically beam scanning in sector $\pm 60^{\circ}$ on both coordinates. Manufacturing of such elements is realized.

2. For $100 \%$ of $3 \cdot 10^{4}$ elements, were measured all main parameters: initial phase, steepness of the linearized PTC and microwave losses. Statistic results shows high manufacturing accuracy - about $80 \%$.

3. Small deviation of initial phases and steepness of PTC for PAA elements lets us to use elements without any information about individual parameters of elements. This factor lets us to design easier scheme of beam control system.

4. Integrated reflective type PAA elements could be used for design of array antennas with different aperture sizes and beam scanning sectors.

\section{References}

1. O.G. Vendik, M.D. Parnes, Electrical Beam Scanning Antennas, 232 pp, (2002)

2. P.S. Hansen, Microvawe beam scanning systems. Volume 2, 496 pp. (1969)

3. N Amitey,V Galindo, Ch. Vu, Phased Array Antennas Theory and Analysis, 457 pp. (1974)

4. P.S. Hansen, Phased Array Antennas 560 pp. (2012)

5. B.V. Bounkin, A.A Lemansky, Experience of Development and Industrial Production of X-Band Passive Phased Antenna Aarrays // Intentional Conference on Radar. Paris. 3-6 May. A. 3. Antenna design. P. 20-24. (1994)

6. D.I. Voskresensky, Microvawe Devices and Antennas. Phased Array Antennas Design. 744 pp. (2012)

7. Yu.N. Afanasev, V.V. Zhigarev, K.n. Zakharev, V.A. Kashin, V,M, Koretsky, A.A. Lemansky, Patent 2184410 Russia. Receiving-Transmitting Phased Array Antenna Element (2002)

8. O. Yu. Shevtsov, A.V. Artyuschev, V.M. Krekhtunov, M.E. Golubtsov, Yu.S. Rusov, Features of Phased Array Antennas for Small-Distance Radar Systems Design. 3. P. 61-69 (2010).

9. L.B. Roshal, A.I. Firsenkov, V.M. Krekhtunov, O. Yu. Shevtsov, Patent 2325741 Russia. Phased Array Antenna Element (2008)

10. A.A. Vasin, A.I. Kanaschenkov, V.M. Krekhtunov, L.I. Ponomarev, A.S. Smirnov, O.V. Terekhin, A.I. Firsenkov, A.A. Firsenkov, Patent 187274 Russia. Pass Type Phased Array Antenna Element (2019)

11. V.V. Denisenko, Yu.B. Dubov, Yu.B. Korchemkin, V.A. Makota, A.I. Nikolaev, A.A. Tolkachev, A.M. Shitikov, A.V. Shishlov, A.T. Shubov, Multielement Ka-band PAA. Antennas. 1 (92). P. 7-14 (2005)

12. A.A. Tolkachev, B.A. Levitan, B.A. Remizov, V.A. Kolobov, G.V. Markin, A.V. Shishlov, A.T. Shubov, Patent 2048699 Russia. Reflective Type Phased Array Antenna (1995)

13. L.V. Naumenko, E.A. Smirnova, V.V. Fedorov, Patent 37877 Russia. Microwave Ferrite Phase Shifter for Reflective Type PAA (2004)

14. V.P. Klapov, B.D. Kozhichenkov, V.D. Korolkov, E.V. Manaenkov, Y.G. Milevsky, Patent 119548 Russia. Microwave Reflective Type Ferrite Phase Shifter (2012)

15. M.D. Parnes, Reflective Array Antenna with Electronic Beam Scanning. Microwave Electronic. 2. P. 24-31 (2019) 
16. S.I. Skovorodnikov, A.B. Guskov, G.D. Pavlov, A.I. Firsenkov, Patent 2470426 Russia. Phased Array Antenna Element (2012)

17. E.V. Komissarova, V.M. Krekhtunov, Patent 2474018 Russia. Phased Reflective Array Antenna Element (2013)

18. A.I. Firsenkov, V.M. Krekhtunov, A.B. Guskov, G.D. Pavlov, Yu.S. Rusov, Patent 2439759 Russia. Phased Array Antenna Element (2010)

19. Phase shifters. http://www.magneton.ru/cat.php?id=105\#main_top (2019)

20. Microwave Ferrites and Dielectrics: http://www.magneton.ru/cat.php?id=32 (2019) 